\title{
Design and Construction of an Optimum Wave Flume
}

\author{
M. R. Khalilabadi ${ }^{1 \dagger}$ and A. A. Bidokhti ${ }^{2}$ \\ ${ }^{1}$ Malek- ashtar University of Technology, Shiraz, Iran \\ ${ }^{2}$ Institute of Geophysics, University of Tehran, Tehran, Iran \\ $\uparrow$ Corresponding Author Email: khalilabadi@ hotmail.com
}

(Received September 25, 2010; accepted April 4, 2011)

\begin{abstract}
This paper describes the design, construction, and testing of a small wave flume and associated equipment. The wave flume is equipped with a flap-type wave generator, capable of producing both regular and irregular waves. Flap paddle is used to examine deep water and floating structures. Instrumentation includes three capacitive wave gauges. The wave-maker is controlled by a desktop computer, which also provides data-logging capability. In order to design a wave absorber of restricted length, all the different mechanisms able to dissipate wave energy studied. Finally from the survey of the wave absorbers used in different laboratories, a gravel beach designed for absorbing the waves coming from wave maker. By sealing leaks around paddle the error of measuring height-to-stroke ratios versus relative depth is decreased by $3 \%$. The main advantage of this system over the previous ones is reduction of the amount of effort in the set up with optimum deflection and low level cost utility.
\end{abstract}

Keywords: Wave tank, Wave-maker, Flap, Deep water, Wave absorber.

\section{NOMENCLATURE}

$\begin{array}{ll}H & \text { Wave height } \\ h & \text { Water depth } \\ \mathrm{k} & \text { Wave number } \\ 1 & \text { Elevation of hinged point from bottom } \\ m_{l} & \text { Height to stroke }\end{array}$

\section{INTRODUCTION}

The use of physical models in marine engineering would be severally limited if we were unable to create waves in small scale models that exhibited many of characteristics of waves in nature. A far more common approach is mechanical wave generation where a movable partition is placed in the wave facility and waves are generated by oscillation of the partition.

So far most laboratory testing of floating or bottommounted structures and studies of wave profiles and other related phenomena have utilized wave flumes, which are usually characterized as long, narrow enclosures with a wave-maker of some kind at one end. For all of these tests, the type of wave-maker is very important. The wave motion that it induces can be determined reasonably well from linear wave theory.

In this paper the design and construction of a small wave flume, which was built and instrumented with a limited budget, is described. This flume was initially constructed as a towing tank in the marine engineering laboratory at Ocean Research Center of Shiraz.

\author{
$S \quad$ Stroke \\ $t \quad$ Time \\ $X(t) \quad$ Wave board displacement \\ $\sigma \quad$ Wave-maker frequency
}

In order to design the wave flume we have reviewed several reports by many designers (e.g. Thomas 2004 \& D2.3 Report 2008), finally regarding our circumstances (funding, time, etc.) we designed the best one that is described in this paper. The main advantage of our system over the previous ones is cost reduction and small effort in system setup.

\section{THEORETICAL APPROACH}

To generate small amplitude, sinusoidal waves with a desired period and wave height, the wave height-tostroke ratio for variable-draft wave-maker is given by (Hughes et al. 1993):

$\frac{H}{S_{0}}=\frac{4 \sinh k h}{\sinh 2 k h+2 k h}\left[\sinh k h+\frac{(\cosh k l-\cosh k h)}{k(h-l)}\right]$

Where $\mathrm{H}$ is the wave height, $\mathrm{k}$ is the wave number, and $h$ is the water depth. Equation (1) is derived from linear wave-maker theory as presented, for example, in Dean and Dalrymple (1991).

Also an expression for the wave board motion is given by (Madsen 1971): 


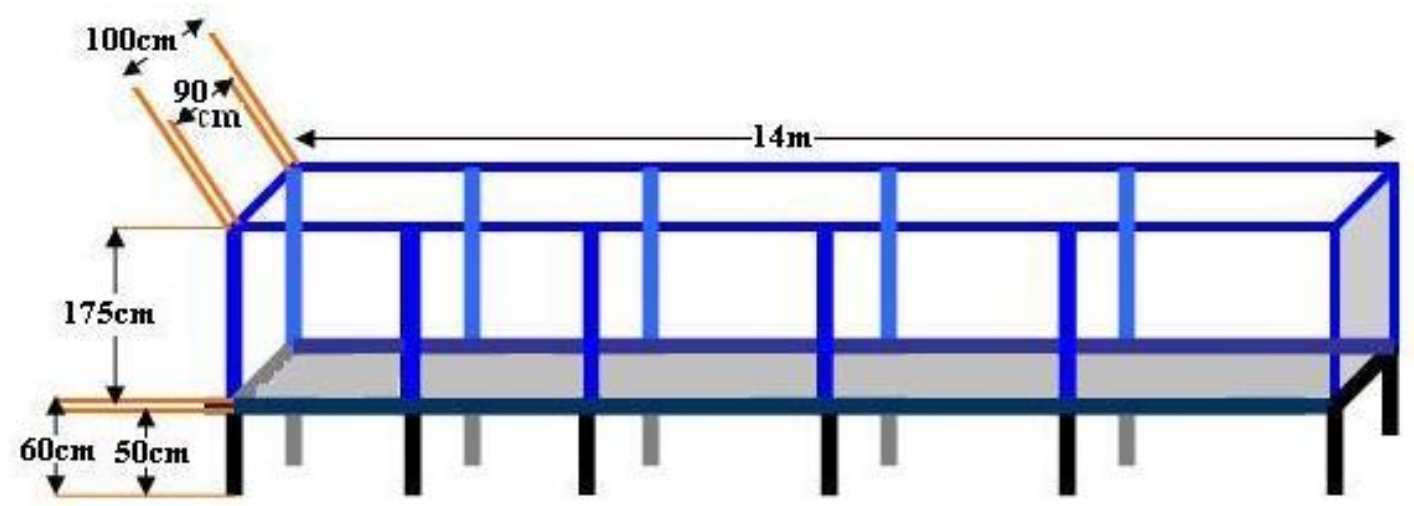

Fig. 1. 3D view of wave flume design

$$
\begin{aligned}
X(t)=\frac{H}{2 m_{l}} \sin \sigma t & +\frac{H^{2}}{32 h\left(1-\frac{h}{2(h+l)}\right)}\left(\frac{3 \cosh k h}{\sinh ^{3} k h}\right. \\
& \left.-\frac{2}{m_{l}}\right) \sin 2 \sigma t
\end{aligned}
$$

Where $m_{l}=\frac{H}{S_{0}}$ is given by Eq. (1) $t$ is the time, $l$ is the elevation of hinged point from bottom, and $\sigma$ is the wave-maker frequency.

\section{DESIGN AND CONSTRUCTION}

The wave flume was built by the Ocean Research Center of Shiraz for conducting laboratory tests of floating structures in the Marine Engineering laboratory. This wave flume design includes a 14-m long, 1.75-m deep, and $1-\mathrm{m}$ wide channel. The bottom of the flume is constructed of aluminum plate of $10-\mathrm{cm}$ thickness. The sides of the flume are made of 5-cm-thick clear glass sheeting, which are supported by two aluminum structural frames. The tank rested on an iron base mounted on 50-cm-height legs. The frame of the final wave flume design is sketched in Fig. 1.

An aquarium pump draws water from one end of the tank and pumps it through a 5-micron residential-type water filter, and then discharges it at the opposite end of the tank. All piping is $25-\mathrm{mm}$ polyvinyl chloride (PVC) with cemented fittings. The intake and discharge pipes can be easily removed for testing to eliminate reflection and diffraction affects.

\subsection{Wave-Maker Design}

A flap-type wave-maker at one end of the tank can generate regular and irregular waves. The flap-paddle is an acrylic plate which hinged on a sill a distance $0.5-\mathrm{m}$ above the bottom of wave flume. The dimensions of paddle is $90-\mathrm{cm}$ wide, $1.25-\mathrm{m}$ high, and $1.3-\mathrm{cm}$ thick. When installed in the tank, it maintains a clearance of 1 $\mathrm{mm}$ along each side. The paddle is driven by an electric servo drive motor. To control the wave-maker, the controller requires information about the hydrodynamic force and paddle position. Position is sensed using the brushless motor resolver on the motor shaft. The force on the paddle is measured with a sealed stainless steel load cell integrated into a sector above the paddle. Force from the air bellow is measured with a single pressure transducer. To generate waves the system uses the central PC streams packets of data to the control cards. Each card stores the drive information and compares the demand with the waves measured on the front face of the paddle. The controller is tuned to run as the complex conjugate of the paddle added mass and damping so as to move and absorb unwanted waves and to generate the demanded ones.

The installed wave-maker is capable of generating regular waves from 0.3-s up to 3-s period and irregular waves from 0.6 -s up to 1.5 -s period. The steepness ratio (height/length) could be varied from 0.05 up to 0.14 for the wave of $3.5-\mathrm{m}$ length. These specifications allow testing the high speed water crafts. Furthermore, the huge range of steepness ratio variation allows investigating the linearity i.e. non-linear phenomenon as very important subject of research for increasing ship safety (Begovic et al. 2006). The wave-maker generates Irregular waves by superposition of hundreds of sinusoidal waves with different wavelengths. The entire standard wave spectrum (ITTC, ISSC, Pierson Moskowitz, JONSWAP, Ochi, and Neumann) can be generated as well as user defined spectrum.

\subsection{Wave Absorber Design}

After the wave generator, wave absorber is the most important part in a wave flume or basin. A great variety of designs and materials have been used throughout the world for the construction of wave absorbers. Wave absorbers could be classified into two main categories: active and passive absorbers. However the use of active absorbers owning to its high cost is still very limited, except in a few cases where the wave board itself is programmed to absorb the reflected wave. For passive absorbers, the beach of constant slop reaching the bottom using sand, gravel or stones seems to be the most popular arrangement, but the use of other materials such as transversal bars; horsehair and wire screen is also popular.

The slope of these absorbers has to be mild so as to obtain a good dissipation of wave energy. This usually means a long wave absorber, thus using up valuable tank space (Dalrymple et al. 2002). In order to reduce the length of the absorber, different arrangements, including the addition of roughness on the surface and the use of porous materials, have been tried. 


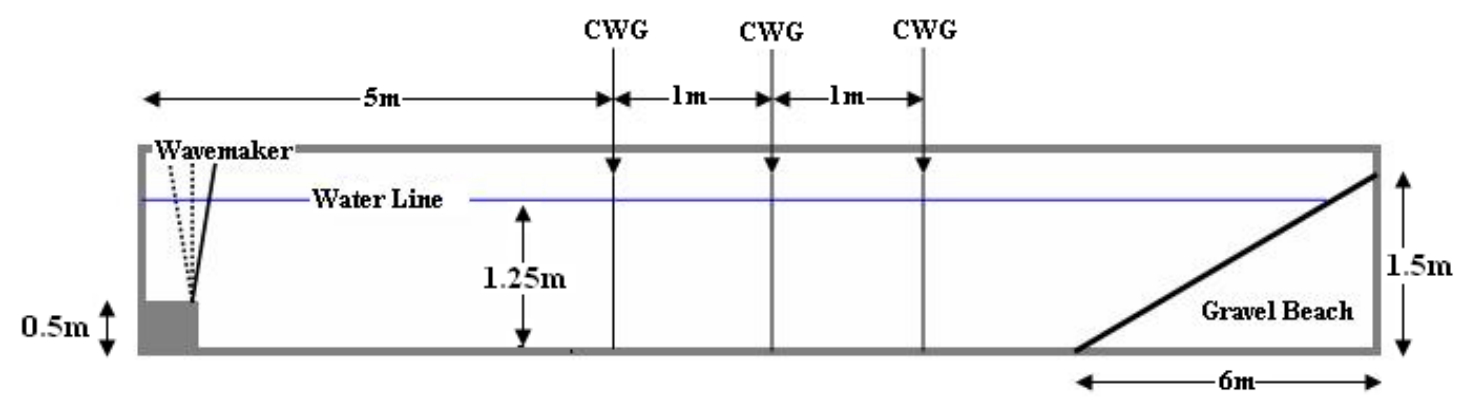

Fig. 2. The configuration of wave gauges and other equipments in the wave flume.

In this project a survey of laboratory facilities around the world has been conducted in order to find an optimum wave absorber satisfying the constraints mentioned above. From 43 laboratories which investigated in this study only four laboratories use active absorbers. Most other laboratories use passive absorbers. Passive absorbers are mainly made up of beaches of constant or varying slopes. The material which constitutes the slop could be permeable. The beaches are often covered by many kinds of rough materials. Some laboratories are using cages filled with different porous materials. One of the important criteria to be satisfied is that the variation of the water depth over a wavelength is small, because abrupt changes of the bottom profile lead to reflection. From 43 laboratories which use passive absorbers, 27 of them use a beach of constant slope reaching the bottom as wave absorber, 7 of them use a variation of this type of wave absorber as a mean of absorbing wave energy, 4 of them use a parabola beach reaching the tank bottom, and 3 of them use a parabola not reaching the bottom. The last two laboratories use a combination of different mechanisms to absorber wave energy. It is clear that most wave flumes tend to use simpler types of absorber shapes.

Among the materials used to roughen surface or to increase porosity (Table 1 ), there is a wide variety, the majority being sand/gravel/stones (22\%), transversal bars (18\%), wire screens (15\%), and horsehair (14\%). About a third of laboratories use some kind of permeable structures. One of the main parameters to be considered is the ratio of the absorbers length to the water depth. Most absorbers have slopes lower than 1:5. In the present work, the slope of absorber was selected 1:4 because of dimensions of the wave flume.

Passive wave absorbers are placed on the basin termination opposite to the wave maker. In this case a gravel beach with a 1:4 slope is located at the end of the tank opposite to the wave-maker. This beach absorbs wave energy both by causing the incident waves to break, and by allowing percolation of water through the gravel after breaking to minimize reflection. The beach is 6-m long and $90-\mathrm{cm}$ wide, and rises from the tank bottom to a maximum depth of 1.5-m (Fig. 2).

\subsection{Wave Gauges and Data Acquisition}

The most common wave gauges are the twin-wire resistance wave gauge and the single-wire capacitance wave gauge (Markle and Greer 1992). These instruments are mounted so that the wire(s) are vertical and piercing the water surface to a depth below the lowest wave trough (i.e. some portion of the wire must remain submerged at all times).

Table 1 Different materials placed over or underneath the wave absorber

\begin{tabular}{|c|c|c|}
\hline \multicolumn{2}{|c|}{ Material } & \multirow{2}{*}{ 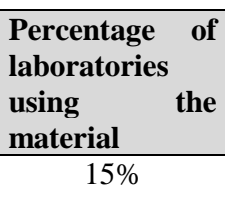 } \\
\hline 1 & Wire screen & \\
\hline 2 & Transversal bars & $18 \%$ \\
\hline 3 & Horse hair & $14 \%$ \\
\hline 4 & $\begin{array}{l}\text { Sand, Gravel and } \\
\text { stones }\end{array}$ & $22 \%$ \\
\hline 5 & Ripples & $5 \%$ \\
\hline 6 & Wooden laths & $5 \%$ \\
\hline 7 & Perforated plywood & $5 \%$ \\
\hline 8 & $\begin{array}{l}\text { Cages filled with } \\
\text { porous materials }\end{array}$ & $10 \%$ \\
\hline 9 & $\begin{array}{l}\text { Group of stainless } \\
\text { pieces }\end{array}$ & $3 \%$ \\
\hline 10 & $\begin{array}{l}\text { Plastic impregnated } \\
\text { cocos fiber (Terrafix) }\end{array}$ & $1.5 \%$ \\
\hline 11 & Concrete & $1.5 \%$ \\
\hline
\end{tabular}

Three capacitive wave gauges are used to measure water surface elevation in the present wave flume. Wave features in the propagation area are determined with capacitance wave gauges (CWG). Three CWGs are positioned along the channel with one meter spacing. They are model WG-50 gauges, manufactured by Richard Brancker Research, Ltd., of Ottawa, Ontario, Canada. Capacitive wave gauges measure the change in electrical discharge time of an immersed, insulated wire. This measured capacitance is linearly proportional to the immersion depth of the wire, and is output by the gauge as a $\pm 5.0 \mathrm{v}$ signal. The gauges may be easily positioned in the tank as needed. Figure 3 shows the configuration of wave gauges and other equipments in the wave flume.

The gauges are calibrated by adjusting two trimmer potentiometers. The first potentiometer sets the zero point for the gauge, and the second potentiometer controls the gain in units of centimeters per volt (Hudson 2001). 


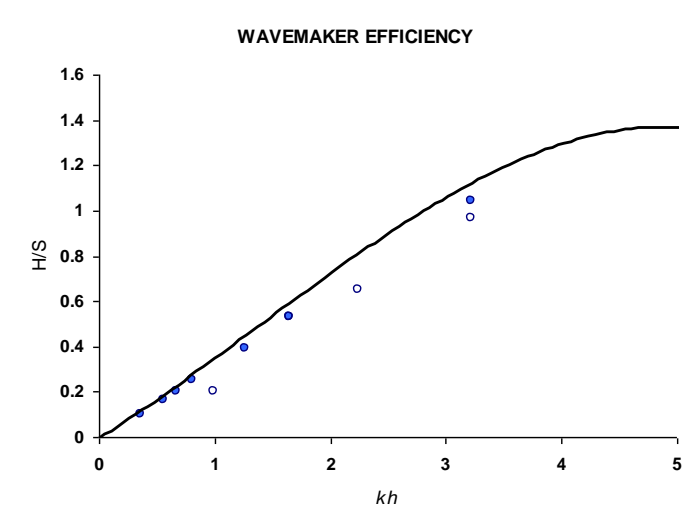

Fig. 3. Experimental wave height $(H)$ to stroke $(S)$ ratio plotted against relative depth $(k h)$. The solid line represents the theoretical ratio, while the open and solid circles are experimental data before and after sealing leaks around paddle respectively

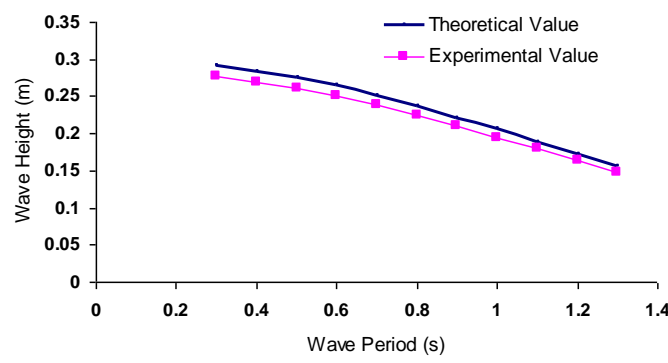

Fig. 4. Experimental and theoretical wave-maker characteristic for regular waves.

Once calibrated, the gauges can operate for several weeks without significant drift. The voltages output from the wave gauges are converted to integer values by an analog-to-digital converter card located in a desktop computer.

During routine operation the wire should be cleaned occasionally to remove any accumulated film which may change the capacitance of the wire or cause the water to adhere to insulation (Kraemer 2001).

The two main advantages of the single-wire capacitance wave gauge are:

a) The gauge exhibits good linearity and dynamic response over a reasonable length so that it can be used for fairly large waves.

b) The gauge is stable over sufficiently long times (gauge "drift" is not a significant problem).

c) Minor obstruction to the wave front.

d) No distortion of the wave shape

e) Low construction costs.

\section{Wave-Maker Performance}

In order to quantify the performance of the linear wavemaker system, waves were generated while there was no model in the tank. Some wave trains were generated for each of combinations of wave height, period, and water depth. For each period, the number of individual waves per train was limited so that the reflected waves did not interfere with the incident waves. The resulting wave height-to-stroke ratios from each of these generated wave tests are plotted against the relative depth in Fig. 4. The no dimensional relative depth is the wave number, $k$, multiplied by the water depth, $h$. in this figure; open and solid circles are experimental data respectively before and after sealing leaks around paddle. The measured wave height-to-stroke ratios in these wave tests before sealing leaks around paddle were approximately $6 \%$ lower than those predicted by wavemaker theory. These ratios, after sealing the leaks around paddle, were approximately $3 \%$ lower than those predicted by wave-maker theory that is a good improvement of the performance of the wave maker.

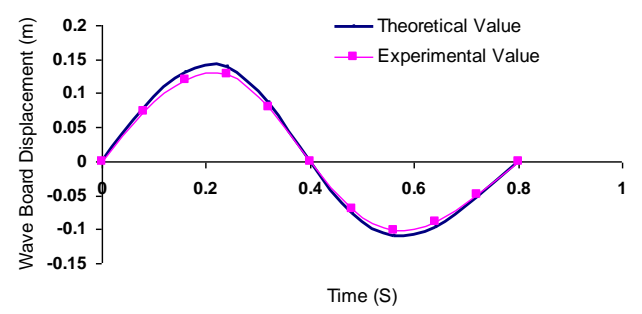

Fig. 5. Experimental and theoretical wave board displacement for generating a wave of about 1-m length

Figure 5 shows the experimental and theoretical wave board displacements required for this wave-maker to generate a wave of about $1-\mathrm{m}$ length. All the tests were done in a constant water depth of $1.25-\mathrm{m}$ and wave board hinged on a sill a distance $0.5-\mathrm{m}$ above the bottom of wave flume.

\section{Conclusion}

The wave flume described in this paper, designed for a specific study and built with a limited budget. a specific study and built with a limited budget. Although this wave flume is small compared to most wave flumes, it is well-suited to educational and basic research studies. Larger flumes require a staff and funding to operate and maintain them, and these overhead costs often prohibit their use in small-scale experiments. Instead of reserving a period of time well in advance to conduct experiments, the researchers using this small flume only need to change it easily to investigate a physical behavior or try out an idea. These initial studies can then be refined and eventually expanded to more complex experiments in large wave tanks or flumes.

In order to absorb waves coming from wave maker in a typical wave flume of restricted dimensions, the choice of a passive absorber is selected. In this wave flume, a gravel beach with a 1:4 slope is located at the end of the tank opposite the wave-maker. This beach absorbs wave energy both by causing the incident waves to break, and by allowing percolation of water through the gravel after breaking to minimize reflection. The measured wave height-to-stroke ratios in experimental tests before sealing leaks around paddle were approximately $6 \%$ lower than those predicted by wave-maker theory while these ratios after sealing the leaks around paddle are approximately $3 \%$ lower. 
The resulting wave heights from generated wave tests were approximately $4.5 \%$ less than theoretical values. That means the average percentage deviation is $4.5 \%$ which is within the experimental error involved in the present laboratory measurements of wave height, period and wave maker stroke. It therefore indicates a good agreement between theory and experiment. In spite of this deflection which is much lower than previous ones (e.g. Thomas 2004 \& D2.3 Report 2008), the sources of this deflection could be mentioned as follow:

- Instrumental Error of wave gages.

- There is a minimum reflection from the end of channel because the dimensions of flume are restricted and we cannot increase the length of wave absorber.

\section{REFERENCES}

Begovic, E., Boccadamo G., Bove A., Caldarella S. (2006). Prismatic hull tests in regular waves in DIN towing tank. Scientific Report, The Naval Engineering Department of University of Naples Federico II, Italy.

Chen, H.C., K. Yu and S.Y. Chen (2004). Simulation of wave run-up around offshore structures by a chimera domain decomposition approach, Civil Engineering in the Oceans VI Conference, Baltimore, Maryland, October 20-22.

Dalrymple, R.A. and Svendsen Ib.A. (2002). Experimental study of near shore dynamics on a barred beach with rip channels. Journal of Geophysical Research 107(C6), 10.1029/2001JC000955.

Dean, R.G., R.A. Dalrymple (1992). Water Wave Mechanics for Engineers and Scientists. World Scientific Publishing Co. Pte. Ltd.

Hudson, P.J. (2001). Wave-Induced Migration of Grounded Ships. Doctoral Dissertation, Johns Hopkins University, 212.

Hughes, S.A. (1993). Physical Models and Laboratory Techniques in Coastal Engineering, World Scientific Publishing Co. Pte. Ltd., China.

Kraemer, D., McCormick M.E., Hudson P.J. and Noble W. (2001). Analysis of the Added-Mass of a Barge in Restricted Waters. Final Report - Phase 2, U.S. Army Corps of Engineers.

Shaffer, H.A. and Steenberg C.M. (2003). Second order wave generating theory for multidirectional waves. Ocean Engineering 30, 1203-1231.

Thomas, O.H. (2004). Evaluation of the Wave Attenuation Characteristics of Three Vertical Slat Breakwaters. Davidson Laboratory, Technical Report SIT-X-XX-XXX, Prepared for: Ocean County, New Jersey Planning Office By: NJ
Coastal Protection Technical Assistance Service. Ship Design and Research Centre S.A. (CTO S.A.) (2008). On the Use of New and Existing Network for European Maritime Research and Testing Facilities. D2.3 Report. 